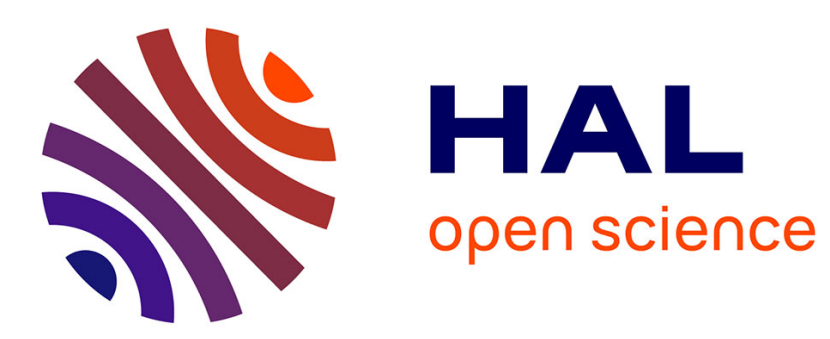

\title{
Analysis of a two-level Schwarz method with coarse spaces based on local Dirichlet-to-Neumann maps
}

Victorita Dolean, Frédéric Nataf, Robert Scheichl, Nicole Spillane

\section{To cite this version:}

Victorita Dolean, Frédéric Nataf, Robert Scheichl, Nicole Spillane. Analysis of a two-level Schwarz method with coarse spaces based on local Dirichlet-to-Neumann maps. Computational Methods in Applied Mathematics, 2012, 12 (4), pp.391-414. hal-00586246

\section{HAL Id: hal-00586246 \\ https://hal.science/hal-00586246}

Submitted on 15 Apr 2011

HAL is a multi-disciplinary open access archive for the deposit and dissemination of scientific research documents, whether they are published or not. The documents may come from teaching and research institutions in France or abroad, or from public or private research centers.
L'archive ouverte pluridisciplinaire HAL, est destinée au dépôt et à la diffusion de documents scientifiques de niveau recherche, publiés ou non, émanant des établissements d'enseignement et de recherche français ou étrangers, des laboratoires publics ou privés. 


\title{
ANALYSIS OF A TWO-LEVEL SCHWARZ METHOD WITH COARSE SPACES BASED ON LOCAL DIRICHLET-TO-NEUMANN MAPS
}

\author{
VICTORITA DOLEAN, FRÉDÉRIC NATAF, ROBERT SCHEICHL, AND NICOLE SPILLANE
}

\begin{abstract}
Coarse grid correction is a key ingredient in order to have scalable domain decomposition methods. For smooth problems, the theory and practice of such two-level methods is well established, but this is not the case for problems with complicated variation and high contrasts in the coefficients. Stable coarse spaces for high contrast problems are also important purely for approximation purposes, when it is not desirable to resolve all the fine scale variations in the problem. In a previous study, two of the authors introduced a coarse space adapted to highly heterogeneous coefficients using the low frequency modes of the subdomain DtN maps. In this work, we present a rigorous analysis of a two-level overlapping additive Schwarz method (ASM) with this coarse space, which provides an automatic criterion for the number of modes that need to be added per subdomain to obtain a convergence rate of the order of the constant coefficient case. Our method is suitable for parallel implementation and its efficiency is demonstrated by numerical examples on some challenging problems with high heterogeneities for automatic partitionings.
\end{abstract}

\section{INTRODUCTION}

When using an iterative method in a one-level domain decomposition framework, one may encounter a long stagnation or a slow convergence even in the case where physical coefficients are homogeneous: convergence deteriorates when the number of subdomains increases. This is a problem since, to perform well, one needs to have a scalable algorithm, i.e., an algorithm whose convergence rate is weakly dependent on the number of subdomains, see [34] and references therein. In order to achieve scalability, global information on the solution needs to be shared between subdomains. This process takes the form of a coarse space correction leading to a two-level algorithm or preconditioner. The BPS preconditioner introduced by Bramble, Paschiak and Schatz [1] is of this type. We can also mention the two-level overlapping Schwarz and the balancing Neumann-Neumann preconditioner, as well as the FETI algorithm, which have been extensively investigated, see [34] and references therein. The abstract analysis of two-level overlapping Schwarz methods, which we will use, is due to [9]. For symmetric systems the balancing preconditioner was proposed by Mandel [20]. The FETI algorithm was first introduced by [13].

The definition of a two-level preconditioner is closely related to its key ingredient: the choice of an appropriate coarse space. Our goal here is to build such a coarse space in the context of domain decomposition methods for elliptic problems with highly heterogeneous coefficients. When the jumps in the coefficients are across subdomain interfaces or inside the subdomains (and not near their boundaries), classical coarse spaces give good results, see

Date: October 27, 2010-beginning; Today is April 14, 2011.

2000 Mathematics Subject Classification. 65F10, 65N22, 65N30, 65 N55.

Key words and phrases. coarse spaces, overlapping Schwarz method, Dirichlet to Neumann maps, eigenvectors, elliptic problems with large coefficient variation. 
e.g. $[8,21,28,26,6,7]$. However, when the discontinuities are along subdomain interfaces, classical results break down. This is what we work to improve.

In previous work, [23], two of the authors proposed the construction of a coarse subspace, which leads to a two-level method that is observed to be robust with respect to heterogeneous coefficients for fairly arbitrary domain decompositions, e.g. provided by an automatic graph partitioner such as METIS or SCOTCH $[19,5]$. This method was extensively studied from a numerical point of view in [24]. The construction is based on the low-frequency modes associated with the Dirichlet-to-Neumann (DtN) map on each subdomain. After obtaining the eigenvectors associated with the near-kernel components of the DtN operator, we use their harmonic extensions to the whole subdomain to build the coarse grid. With this method, even for discontinuities along (rather than across) the subdomain interfaces, the iteration counts are robust to arbitrarily large jumps of the coefficients leading to a very efficient, automatic method for these kinds of problems. It is also suitable for parallel implementation. Similar ideas to build stable coarse spaces, based on the solution of eigenvalue problems on the whole subdomain, have also been presented in other papers on Schwarz methods [15, 10], as well as in earlier work on algebraic multigrid methods [2, 4].

In this paper we will analyze the two-level preconditioner proposed in [23] theoretically. In order to do this, we will use the framework of weighted Poincaré inequalities, introduced and successfully applied to different domain decomposition methods in [29, 30, 27, 14, 32, 33]. Our analysis is inspired by the techniques in [15], as well as by the abstract framework developed in [33]. However, the DtN coarse space is better designed to deal with coefficient variations that are strictly interior to the subdomain, leading to a smaller dimension than the coarse space proposed in [15]. Only heuristic ideas are given in [15] to overcome this problem. For our coarse space we have a rigorous justification. The result that we obtain, generalizes the classical estimates of overlapping Schwarz methods to the case where the coarse space is richer than just the constant mode per domain [25], or other classical coarse spaces (cf. [34]).

The rest of the paper is organized as follows. In Section 2 we introduce the general framework. The model problem, its discretization and some weighted norms, depending on the coefficients, are introduced in Section 2.1. Then the decomposition into subdomains, the overlapping Schwarz method and some weighted Poincaré inequalities, including a new trace result, are presented in Sections 2.2-2.3. In Section 3 the new coarse space is presented, at local and global level, and theoretical stability results are shown (e.g. stability of the coarse space projectors), then a condition number estimate of the two-level method based on the existence of a stable decomposition is given in Theorem 3.5. Extensive numerical results for rather hard test cases are given in Section 4, followed by some conclusions in Section 5 .

\section{PREliminaries AND NOTATION}

2.1. Model problem and discretization. We consider the variational formulation of a second order, elliptic boundary value problem with Dirichlet boundary conditions: Find $u^{*} \in H_{0}^{1}(\Omega)$, for a given polygonal (polyhedral) domain $\Omega \subset \mathbb{R}^{d}(d=2$ or 3 ) and a source term $f \in L_{2}(\Omega)$, such that

$$
\underbrace{\int_{\Omega} \alpha(\boldsymbol{x}) \nabla u^{*} \cdot \nabla v}_{\equiv a\left(u^{*}, v\right)}=\underbrace{\int_{\Omega} f(\boldsymbol{x}) v(\boldsymbol{x})}_{\equiv(f, v)}, \text { for all } v \in H_{0}^{1}(\Omega) .
$$




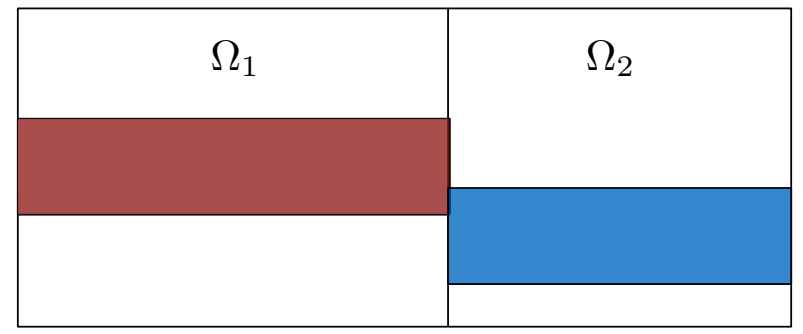

Figure 1. Coefficient $\alpha$ varying along and across the interface.

We are interested in the case where the diffusion coefficient $\alpha=\alpha(\boldsymbol{x})$ is a positive piecewise constant function that may have large variations within $\Omega$, especially with discontinuities along and across the subdomain interfaces (see Figure 1). To be precise, we assume that the domain is a union of polygonal (polyhedral) subdomains $\mathcal{Y}_{l}$, such that:

$$
\bar{\Omega}=\bigcup_{l=1}^{m} \overline{\mathcal{Y}}_{l} \text { and } \alpha(\boldsymbol{x})=\alpha_{l}, \text { for all } \boldsymbol{x} \in \mathcal{Y}_{l} \text { and } l=1, \ldots, m \text {. }
$$

For any domain $D \subset \Omega$ we need the usual norms, with the standard notations $\|\cdot\|_{L_{2}(D)}$, $|\cdot|_{H^{1}(D)}$ and $\|\cdot\|_{H^{1}(D)}$, as well as the $L_{2}$ inner product $(v, w)_{L_{2}(D)}$. In addition to this, we need to define some related weighted quantities, which will be very useful in the following:

- the weighted $H^{1}$ (or energy) norm

$$
|v|_{a, D}^{2}=\int_{D} \alpha(\boldsymbol{x})|\nabla v|^{2}
$$

Note that if $v \in H_{0}^{1}(D)$ this is indeed a norm, and for $v \in H^{1}(D)$ this is only a seminorm. We denote the seminorm by $|\cdot|_{a, D}$.

- the weighted $L_{2}$ norm

$$
\|v\|_{0, \alpha, D}^{2}=\int_{D} \alpha(\boldsymbol{x}) v^{2}
$$

- the weighted $L_{2}$ inner product

$$
(v, w)_{0, \alpha, D}=\int_{D} \alpha(\boldsymbol{x}) v w .
$$

When $D=\Omega$ we omit the domain from the subscript and write $\|\cdot\|_{a}$ and $\|\cdot\|_{0, \alpha}$ instead of $\|\cdot\|_{a, \Omega}$ and $\|\cdot\|_{0, \alpha, \Omega}$, respectively.

Finally, we will also need averages and norms defined on $(d-1)$-dimensional manifolds $X \subset \mathbb{R}^{d}$, namely for any $v \in L_{2}(X)$ and for any $\beta \in L_{\infty}(X)$ we define

$$
\bar{v}^{X}:=\frac{1}{|X|} \int_{X} u \text { and }\|v\|_{0, \beta, X}:=\int_{X} \beta v^{2} .
$$

We consider a discretization of the variational problem (2.1) with continuous, piecewise linear finite elements (FE). To define the FE spaces and the approximate solution, we assume that we have a shape regular, simplicial triangulation $\mathcal{T}_{h}$ of $\Omega$. We assume that this triangulation also resolves $\mathcal{Y}_{l}$, namely, for $l=1, \ldots, m$ we have:

$$
\bar{\Omega}=\bigcup_{\tau \in \mathcal{T}_{h}} \tau \text { and } \overline{\mathcal{Y}}_{l}=\bigcup_{\tau \in \mathcal{T}_{h, l}} \tau \text {, }
$$


where $\mathcal{T}_{h, l} \subset \mathcal{T}_{h}$, for $l=1, \ldots, m$. The standard space of continuous and piecewise linear (w.r.t $\mathcal{T}_{h}$ ) functions is then denoted by $V_{h}$, and the subspace of functions from $V_{h}$ that vanish on the boundary of $\Omega$ by $V_{h, 0}$. In our analysis, we will also need restrictions of FE functions into subdomains $D \subset \Omega$ that are resolved by $\mathcal{T}_{h}$. The space of restrictions of the functions in $V_{h}$ to $D$ is denoted by $V_{h}(D)$. Similarly, the space of restrictions of functions from $V_{h}$, which are supported in $\bar{D}$ is denoted by $V_{h, 0}(D)$. Thus, $V_{h}(D) \subset H^{1}(D)$ and $V_{h, 0}(D) \subset H_{0}^{1}(D)$. $I_{h}$ denotes the standard nodal value interpolation operator from $C(\bar{D})$ to $V_{h}(D)$.

To finish this section let us write the discrete FE problem that we want to solve: Find $u_{h} \in V_{h, 0}$ such that

$$
a\left(u_{h}, v_{h}\right)=\left(f, v_{h}\right), \quad \text { for all } \quad v_{h} \in V_{h, 0} .
$$

In the description and in the analysis of our preconditioners we will frequently switch between this variational point of view of the problem and a purely algebraic one. For that matter, let $\left\{\phi_{i}\right\}_{i=1}^{n}$ be the usual basis for $V_{h, 0}$ consisting of nodal "hat" functions with $n:=\operatorname{dim}\left(V_{h, 0}\right)$. Then $(2.5)$ can be compactly written as

$$
A \boldsymbol{u}=\boldsymbol{f},
$$

where $A_{i, j}:=a\left(\phi_{j}, \phi_{i}\right), f_{i}=\left(f, \phi_{i}\right), i, j=1, \ldots, n$ and $\boldsymbol{u}$ is the vector of coefficients corresponding to the unknown FE function $u_{h}$ in (2.5). In the following we will often switch between matrices and their corresponding bilinear forms, e.g. $A$ and $a(\cdot, \cdot)$, as well as between coefficient vectors in $\mathbb{R}^{n}$ and their corresponding $\mathrm{FE}$ functions in $V_{h, 0}$, e.g. $\boldsymbol{u}$ and $u_{h}$. We use boldface for vectors and roman for $\mathrm{FE}$ functions.

Throughout the paper, the notation $E \lesssim F$ (for two quantities $E, F$ ) means that $E / F$ is bounded above independently, not only of the mesh size $h$ and the method specific parameters ( $\operatorname{such}$ as $\operatorname{diam}\left(\Omega_{j}\right)$ and $\delta_{j}$, defined below for $\left.j=1, \ldots, J\right)$, but also of the values of the coefficient $\alpha(\boldsymbol{x})$ at all $\boldsymbol{x} \in \Omega$. Moreover $E \approx F$ means that $E \lesssim F$ and $E \lesssim F$.

2.2. Automatic domain decomposition and partitions of unity. In order to automatically construct robust two-level Schwarz type methods for (2.5) we first partition our domain $\Omega$ into a set of non-overlapping subdomains $\left\{\Omega_{j}^{\prime}\right\}_{j=1}^{J}$ using for example a graph partitioner such as METIS or SCOTCH[19, 5]. Each subdomain $\Omega_{j}^{\prime}$ is then extended to a domain $\Omega_{j}$ by adding one or several layers of fine grid elements, thus creating an overlapping decomposition $\left\{\Omega_{j}\right\}_{j=1}^{J}$ of $\Omega$. For the moment we only assume that each point $\boldsymbol{x}$ in $\Omega$ is contained in at most $N_{0}$ subdomains.

We also need a partition of unity of functions $\left\{\chi_{j}\right\}_{j=1}^{J}$ defined on $\Omega$, subordinate to the overlapping decomposition $\left\{\Omega_{j}\right\}_{j=1}^{J}$. To construct such a partition of unity automatically we define first, for each fine grid node $\boldsymbol{x}_{i} \in \Omega$, the index set $\mathcal{N}\left(\boldsymbol{x}_{i}\right)$ that contains the indices of all the domains $\Omega_{j}$ that contain $\boldsymbol{x}_{i}$. Then, for each subdomain $\Omega_{j}$ we define $\chi_{j} \in V_{h, 0}\left(\Omega_{j}\right)$ by setting

$$
\chi_{j}\left(\boldsymbol{x}_{i}\right):=\frac{\operatorname{dist}\left(\boldsymbol{x}_{i}, \partial \Omega_{j}\right)}{\sum_{k \in \mathcal{N}\left(\boldsymbol{x}_{i}\right)} \operatorname{dist}\left(\boldsymbol{x}_{i}, \partial \Omega_{k}\right)}, \quad \text { at all fine grid nodes } \boldsymbol{x}_{i} \in \Omega_{j} .
$$

Clearly these functions form a partition of unity on $\Omega$ and satisfy $0 \leq \chi_{j} \leq 1$. Moreover, if

$$
\Omega_{j}^{\circ}:=\left\{\boldsymbol{x} \in \Omega_{j}: \chi_{j}(\boldsymbol{x})<1\right\}
$$


denotes the boundary layer of $\Omega_{j}$ that is overlapped by neighbouring domains, then it is also easy to verify that

$$
\left|\nabla \chi_{j}\right| \lesssim \delta_{j}^{-1}
$$

where $\delta_{j}$ denotes the width of $\Omega_{j}^{\circ}$ at the narrowest place.

We now state the assumption that links the distribution of the values of the coefficient $\alpha(\boldsymbol{x})$ of the PDE and the domain partitioning.

Assumption 2.1. We assume that each $\Omega_{j}^{\circ}, j=1, \ldots, J$, can be subdivided into regions $D_{j k}, k=1, \ldots, K_{j}$, such that $\operatorname{diam}\left(D_{j k}\right) \approx \delta_{j}$ and $\left|D_{j k}\right| \approx \delta_{j}^{d}$, and such that, for each $k$, there exists a $(d-1)$-dimensional manifold $X_{k} \subset \partial \Omega_{j} \cap \bar{D}_{j k}$ with the following properties: (i) $\left|X_{k}\right| \approx \delta_{j}^{d-1}$, (ii) $\max _{\boldsymbol{x}, \boldsymbol{y} \in X_{k}} \alpha(\boldsymbol{x}) / \alpha(\boldsymbol{y})=\mathcal{O}(1)$ and (iii) there exists a path $P_{\boldsymbol{y}}$ from each $\boldsymbol{y} \in D_{j k}$ to $X_{k}$, such that $\alpha(\boldsymbol{x})$ is an increasing function along $P_{\boldsymbol{y}}$ (from $\boldsymbol{y}$ to $X_{k}$ ). Without loss of generality, we assume that the triangulation $\mathcal{T}_{h}$ resolves each of the regions $D_{j k}$ and each of the manifolds $X_{k}$. See Figure 2 for some typical examples where the assumption is either verified or not verified.

Remark 2.2. Following [29, 30], if condition (iii) in Assumption 2.1 holds, $\alpha(x)$ is called quasi-monotone on $D_{j k}$ with respect to $X_{k}$. It implies that the manifold $X_{k}$ lies in the closure of the region $\mathcal{Y}_{l}$, where $\alpha$ takes its maximum on $D_{j k}$. This quasi-monotonicity is similar to the classical quasi-monotonicity defined in [8], if we can cluster the points in $D_{j k}$ into shape-regular subregions $Y$ of diameter $\approx \delta_{j}$, where the coefficient variation is $\mathcal{O}(1)$, and if we can then find a path $P_{Y}$ with minimum diameter $\gtrsim \delta_{j}$ from $Y$ to $X_{k}$ such that $\alpha(x)$ is an increasing function along $P_{Y}$ (from $Y$ to $X_{k}$ ). If one of these paths $P_{Y}$ is connected only via an $m$-dimensional manifold, $m<d-1$, then we call $\alpha(x)$ type- $m$ quasi-monotone on $D_{j k}$ with respect to $X_{k}$. Assumption 2.1 provides only a sufficient condition for the theory below. It is not a necessary condition and various generalizations and extensions similar to those in [27] could be considered. We will not do this here.

The following two lemmas, based on Assumption 2.1, are crucial in the analysis below.

Lemma 2.3. Let Assumption 2.1 be satisfied. Then there exists a uniform constant $C_{P}>0$ independent of the values of $\alpha(x)$, such that the following weighted Poincaré-type inequality holds for all $j=1, \ldots, J$ and $k=1, \ldots, K_{j}$ :

$$
\left\|v-\bar{v}^{X_{k}}\right\|_{0, \alpha, D_{j k}} \leq C_{P} \delta_{j}|v|_{a, D_{j k}}, \quad \text { for all } v \in V_{h}\left(\mathcal{D}_{j k}\right) .
$$

The constant $C_{P}$ is independent of $h, \delta_{j}$ and $\operatorname{diam}\left(\Omega_{j}\right)$, if $\alpha(x)$ satisfies the classical quasimonotonicity condition (cf. Remark 2.2) on all $D_{j k}$. It depends on $\log \left(\delta_{j} / h\right)\left(\right.$ resp. $\left.\delta_{j} / h\right)$, if $\alpha(x)$ is only type- $(d-2)$ (resp. type- $(d-3)$ ) quasi-monotone on any $D_{j k}, k=1, \ldots, K_{j}$.

Proof. Theorem 2.2 and Theorem 3.3 in [29].

Lemma 2.4. Let Assumption 2.1 be satisfied and $\operatorname{tr}_{j} \alpha(\boldsymbol{x}):=\lim _{\boldsymbol{y} \in \Omega_{j} \rightarrow \boldsymbol{x}} \alpha(\boldsymbol{y})$, for a.e. $\boldsymbol{x} \in \partial \Omega_{j}$. Then

$$
\|u\|_{0, \alpha, \Omega_{j}^{\circ}}^{2} \lesssim C_{P}^{2} \delta_{j}^{2}|u|_{a, \Omega_{j}^{\circ}}^{2}+\delta_{j}\|u\|_{0, \operatorname{tr}_{j} \alpha, \partial \Omega_{j}}^{2}, \quad \text { for all } u \in V_{h}\left(\Omega_{j}^{\circ}\right) .
$$

If $\alpha(x)$ satisfies the classical quasi-monotonicity condition (cf. Remark 2.2) on all $D_{j k}$, then the statement holds for all $u \in H^{1}\left(\Omega_{j}^{\circ}\right)$. 


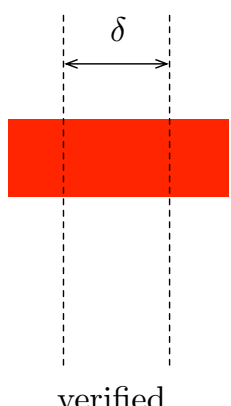

verified

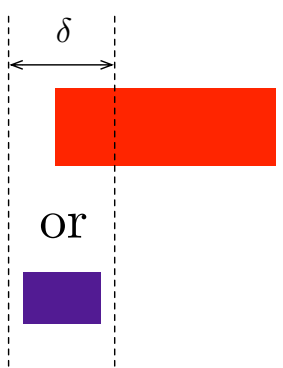

not verified

FigURE 2. Overlap region between two subdomains with high-permeability inclusions, such that Assumption 2.1 is verified (left) and not verified (right).

Proof. Let $\left\{D_{j k}\right\}_{k=1}^{K_{j}}$ be the partitioning of $\Omega_{j}^{\circ}$ in Assumption 2.1 and let $X_{k}$ be the $(d-1)$ dimensional manifold associated with $D_{j k}$. Let $\|\alpha\|_{\infty, D_{j k}}:=\operatorname{esssup}\left\{\alpha(\boldsymbol{x}): \boldsymbol{x} \in D_{j k}\right\}$. Then it follows from Lemma 2.3, as well as the triangle and the Cauchy-Schwarz inequalities, that

$$
\begin{aligned}
\frac{1}{2}\|u\|_{0, \alpha, D_{j k}}^{2} & \leq\left\|u-\bar{u}^{X_{k}}\right\|_{0, \alpha, D_{j k}}^{2}+\left\|\bar{u}^{X_{k}}\right\|_{0, \alpha, D_{j k}}^{2} \\
& \leq C_{P}^{2} \delta_{j}^{2}|u|_{a, D_{j k}}^{2}+\frac{\left|D_{j k}\right|}{\left|X_{k}\right|^{2}}\|\alpha\|_{\infty, D_{j k}}\left(\int_{X_{k}} u\right)^{2} \\
& \leq C_{P}^{2} \delta_{j}^{2}|u|_{a, D_{j k}}^{2}+\frac{\left|D_{j k}\right|}{\left|X_{k}\right|}\|\alpha\|_{\infty, D_{j k}} \int_{X_{k}} u^{2} \\
& \lesssim C_{P}^{2} \delta_{j}^{2}|u|_{a, D_{j k}}^{2}+\delta_{j}\|u\|_{0, \alpha, X_{k}}^{2} .
\end{aligned}
$$

In the last step, we have used Assumption 2.1, i.e. $\left|D_{j k}\right| /\left|X_{k}\right| \lesssim \delta_{j}, \alpha(x)$ attains its maximum on $\bar{D}_{j k}$ in a set containing $X_{k}$, and $\max _{\boldsymbol{x}, \boldsymbol{y} \in X_{k}} \alpha(\boldsymbol{x}) / \alpha(\boldsymbol{y})=\mathcal{O}(1)$. The final result follows by summing over $k=1, \ldots, K_{j}$.

2.3. Two-level overlapping Schwarz method. Having defined overlapping subdomains in Section 2.2, one-level Schwarz-type preconditioners for (2.5) or (2.6) can now simply be introduced by defining restriction operators $R_{j}$ from functions in $V_{h, 0}$ to functions in $V_{h, 0}\left(\Omega_{j}\right)$ or from vectors in $\mathbb{R}^{n}$ to vectors in $\mathbb{R}^{n_{j}}$, where $n_{j}:=\operatorname{dim} V_{h, 0}\left(\Omega_{j}\right)$. As usual we use simple injection, i.e. for any $u \in V_{h, 0}$ we set $\left(R_{j} u\right)\left(\boldsymbol{x}_{i}\right)=u\left(\boldsymbol{x}_{i}\right)$ for every grid node $\boldsymbol{x}_{i} \in \Omega_{j}$. In matrix notation the one-level overlapping additive Schwarz preconditioner is now simply

$$
M_{A S, 1}^{-1}=\sum_{j=1}^{J} R_{j}^{T} A_{j}^{-1} R_{j} \quad \text { where } \quad A_{j}:=R_{j} A R_{j}^{T} .
$$

This preconditioner is particularly well suited for preconditioning parallel iterative solvers, such as conjugate gradients (CG) for (2.6), since all the subdomain solves can be carried out independently of each other. However, the condition number and thus the number of CG iterations grow with the number of subdomains $J$. Thus, it is essential to include a second, coarse level in the preconditioner.

Let us assume that we have a subspace $V_{H} \subset V_{h, 0}$ and a restriction operator $R_{H}$ from $V_{h, 0}$ to $V_{H}$. Then the two-level overlapping additive Schwarz preconditioner can be defined 
similarly to $M_{A S, 1}^{-1}$ as

$$
M_{A S, 2}^{-1}=R_{H}^{T} A_{H}^{-1} R_{H}+\sum_{j=1}^{J} R_{j}^{T} A_{j}^{-1} R_{j} \quad \text { where } \quad A_{H}:=R_{H} A R_{H}^{T} .
$$

In the classical algorithm $V_{H}$ consists simply of FEs on a coarser triangulation $\mathcal{T}_{H}$ of $\Omega$ and $R_{H}$ is the canonical restriction from $V_{h, 0}$ to $V_{H}$, leading to a fully scalable iterative method (provided $\min _{j} \delta_{j} \approx H$ ). However, unfortunately this preconditioner is not fully robust to strong variations in the coefficient $\alpha$ and many recent articles, such as $[16,31,15,33,35]$, have attempted to propose and analyse alternative choices for $V_{H}$ and $R_{H}$ that lead to a fully robust algorithm. In the next section, we will present a new, completely local approach to construct such a coarse space and such a restriction using eigenvectors of local Dirichletto-Neumann maps proposed in [23, 24].

Several alternative versions of these simple additive preconditioners exist, including the multiplicative Schwarz preconditioner, hybrid versions, deflation based coarse grid correction, or the restricted additive Schwarz (RAS) preconditioner [3]. We will only analyse the additive preconditioner, but we note that all the other symmetric versions can be analysed in the same way. The RAS variant is slightly different since it leads to a nonsymmetric iteration, but we will see in Section 4 that it behaves in a similar way and can often give slightly better results than the classical additive version above.

\section{Coarse space construction based on local Dirichlet-to-Neumann maps}

Our coarse space and the restriction operator will be constructed by defining a suitable set of basis functions $\left\{\Phi_{k}\right\}_{k=1}^{N}$ with $N$ usually larger than $J$, such that $V_{H}=\operatorname{span}\left\{\Phi_{k}\right\}_{k=1}^{N}$ and the restriction $R_{H}$ is the canonical restriction associated with this basis. The construction we propose, is to some extent inspired by two observations, already made elsewhere, namely

- that robust coarse space basis functions can in many cases be obtained on standard simplicial meshes by harmonically extending suitable boundary data to the interior of coarse mesh elements [16],

- that local spectral information about the underlying differential operator can be used to obtain fully robust coarse spaces $[15,33]$.

While the coarse spaces in [16] are not able to deal with completely general piecewise constant coefficients, the spaces in [15] are often too large, especially when there are many small, isolated inclusions with large coefficient values. The coarse space presented here suffers from neither of these drawbacks since it is based on local spectral information (so it is parallelizable), and relies on minimal size interface information (only "absolutely necessary" modes are taken into account in the coarse space). This interface information is then further extended harmonically to the interior of the subdomains.

3.1. Local coarse space construction. To build a coarse space $V_{H}$ for (2.5) we will first construct local basis functions on each subdomain $\Omega_{j}$. To this end, let us fix $j \in\{1, \ldots, J\}$ and first consider at the continuous level the Dirichlet-to-Neumann map $\operatorname{DtN}_{j}$ on the boundary of $\Omega_{j}$. Let $\Gamma:=\partial \Omega_{j}$ and let $v_{\Gamma}: \Gamma \rightarrow \mathbb{R}$ (such that $\left.v_{\Gamma}\right|_{\partial \Omega}=0$ if $\left.\Gamma \cap \partial \Omega \neq \emptyset\right)$. We define

$$
\operatorname{DtN}_{j}\left(v_{\Gamma}\right):=\left.\alpha \frac{\partial v}{\partial \boldsymbol{n}_{j}}\right|_{\Gamma}
$$




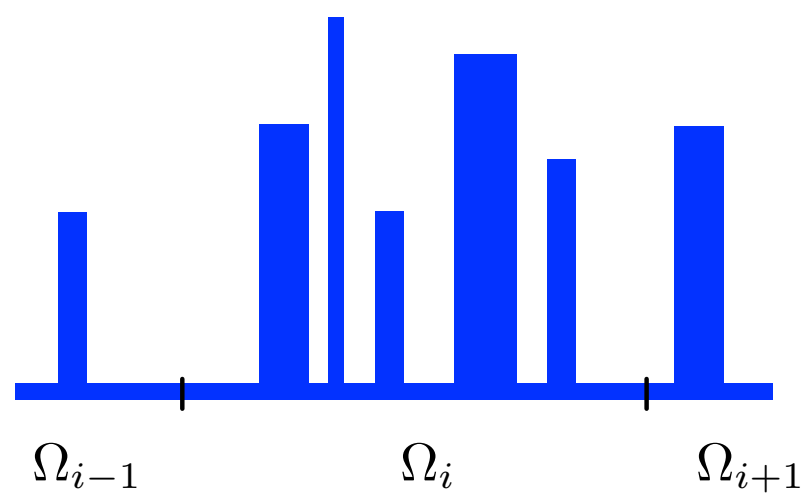

FigURE 3. 1D example with many high coefficient inclusions per subdomain.

where $\boldsymbol{n}_{j}$ is the unit outward normal to $\Omega_{j}$ on $\Gamma$, and $v$ satisfies

$$
\begin{aligned}
-\operatorname{div}(\alpha \nabla v) & =0 & & \text { in } \Omega_{j}, \\
v & =v_{\Gamma} & & \text { on } \Gamma .
\end{aligned}
$$

We see that $\operatorname{DtN}_{j}$ maps the Dirichlet data $v_{\Gamma}$ on $\Gamma$ to Neumann data (fluxes) on $\Gamma$. The function $v$ is the $\alpha$-harmonic extension of the boundary data $v_{\Gamma}$ to the interior of $\Omega_{j}$. To construct the (local) coarse basis functions, we use the low frequency modes of the Dirichlet to Neumann operator $\mathrm{DtN}_{j}$ with respect to the weighted $L_{2}$-norm on $\Gamma$, i.e. the smallest eigenvalues of

$$
\operatorname{DtN}_{j}\left(v_{\Gamma}\right)=\lambda \alpha v_{\Gamma}
$$

For simplicity let us consider an interior subdomain $\Omega_{j}$ that does not touch the (Dirichlet) boundary of the global domain $\Omega$. The other case carries through in a similar way.

Instead of looking for an eigenpair of equation (3.2) and then compute $v$ the harmonic extension of $v_{\Gamma}$, we directly search for the pair $(v, \lambda)$. It is straightforward to check that it satisfies:

$$
\begin{array}{rlrl}
-\operatorname{div}(\alpha \nabla v) & =0 & \text { in } \Omega_{j}, \\
\alpha \frac{\partial v}{\partial \boldsymbol{n}_{j}} & =\lambda \alpha v & & \text { on } \Gamma .
\end{array}
$$

The variational formulation of $(3.3)$ is: Find $(v, \lambda) \in H^{1}\left(\Omega_{j}\right) \times \mathbb{R}$ such that

$$
\int_{\Omega_{j}} \alpha \nabla v \cdot \nabla w=\lambda \int_{\Gamma} \operatorname{tr}_{j} \alpha v w, \quad \forall w \in H^{1}\left(\Omega_{j}\right)
$$

where $\operatorname{tr}_{j} \alpha$ is as defined in Lemma 2.4.

Remark 3.1. We note a clear relationship with the work of Galvis and Efendiev [15]. However, the significant difference lies in the fact that the latter uses local spectral basis functions associated with the eigenproblem $-\operatorname{div}(\alpha \nabla v)=\lambda \alpha v$, posed on the entire subdomain $\Omega_{j}$, which will always lead to a larger number of potentially critical eigenmodes. We can easily illustrate this using the one-dimensional example in Figure 3: whatever the number of islands in the interior of $\Omega_{j}$, our DtN coarse space consists of two vectors per subdomain at most, since the DtN map is a two by two matrix which has two eigenvectors. 
To obtain the discrete form of the generalized eigenvalue problem (3.4), we consider the bilinear forms $a_{j}: H^{1}\left(\Omega_{j}\right) \times H^{1}\left(\Omega_{j}\right) \rightarrow \mathbb{R}$

$$
a_{j}(v, w):=\int_{\Omega_{j}} \alpha \nabla v \cdot \nabla w \text { and } m(v, w):=\int_{\Gamma} \operatorname{tr}_{j} \alpha v w, \quad \forall w \in H^{1}\left(\Omega_{j}\right) .
$$

With a finite element basis $\left\{\phi_{k}\right\}$, the coefficient matrix of the variational form $a_{j}$ is

$$
A_{k l}^{(j)}=\int_{\Omega_{j}} \alpha \nabla \phi_{k} \cdot \nabla \phi_{l} .
$$

Note that, for the whole domain $\Omega$, the coefficient matrix is given by

$$
A_{k l}=\int_{\Omega} \alpha \nabla \phi_{k} \cdot \nabla \phi_{l}
$$

Let I (resp. $\Gamma$ ) be the set of indices corresponding to the interior (resp. boundary) degrees of freedom and $n_{\Gamma}:=\# \Gamma$ the number of interface degrees of freedom. With block notations, we have

$$
A_{\mathrm{II}}^{(j)}=A_{\mathrm{II}}, \quad A_{\Gamma \mathrm{I}}^{(j)}=A_{\Gamma \mathrm{I}} \quad \text { and } \quad A_{\mathrm{I} \Gamma}^{(j)}=A_{\mathrm{I} \Gamma} .
$$

The matrix $A_{\Gamma \Gamma}^{(j)} \neq A_{\Gamma \Gamma}$, since it refers to the matrix prior to assembly with the neighboring subdomains. Therefore, it cannot simply be extracted from the coefficient matrix $A$.

Let $M$ be the weighted mass matrix on $\Gamma$ corresponding to the variational form $m$ i.e.,

$$
(M)_{k l}:=\int_{\Gamma} \operatorname{tr}_{j} \alpha \phi_{k} \phi_{l}
$$

Then we can write down the finite element approximation to generalized eigenproblem (3.4) in matrix notation

$$
A^{(j)} \mathbf{V}=\lambda M \mathbf{V}
$$

where $\mathbf{V}$ denotes the degrees of freedom of the finite element approximation to $v$.

To define the discrete action of the DtN map, let now $v_{\Gamma, h}:=\sum_{k \in \Gamma} V_{\Gamma, k} \phi_{k}$ be some predescribed piecewise linear boundary data on $\Gamma$ with coefficient vector $\mathbf{V}_{\Gamma} \in \mathbb{R}^{n_{\Gamma}}$, and let $V_{h}:=\sum_{k \in \mathrm{I}} V_{\mathrm{I}, k} \phi_{k}+\sum_{l \in \Gamma} V_{\Gamma, l} \phi_{l} \in V_{h}\left(\Omega_{j}\right)$ be its discrete harmonic extension. In matrix notation, the finite element approximation top (3.1) reads $A_{\mathrm{II}} \mathbf{U}_{\mathrm{I}}+A_{\mathrm{I} \Gamma} \mathbf{U}_{\Gamma}=\mathbf{0}$, leading to the usual discrete $\alpha$-harmonic extension

$$
\mathbf{U}_{\mathrm{I}}=-A_{\mathrm{II}}^{-1} A_{\mathrm{I} \Gamma} \mathbf{U}_{\Gamma}
$$

We introduce

Thus we have,

$$
\left(M_{\Gamma}\right)_{k l}:=\int_{\Gamma} \operatorname{tr}_{j} \alpha \phi_{k} \phi_{l}, \quad \text { for all } k, l \in \Gamma
$$

$$
A_{\Gamma \Gamma}^{(j)} \mathbf{V}_{\Gamma}+A_{\Gamma \mathrm{I}} \mathbf{V}_{\mathrm{I}}=\lambda M_{\Gamma} \mathbf{V}_{\Gamma}
$$

and using the discrete $\alpha$-harmonic extension (3.6), the discrete form of (3.2) is a generalised eigenvalue problem

$$
S_{\Gamma} \mathbf{V}_{\Gamma}=\lambda M_{\Gamma} \mathbf{V}_{\Gamma} .
$$

for the Schur complement $S_{\Gamma}:=A_{\Gamma \Gamma}^{(j)}-A_{\Gamma \mathrm{I}} A_{\mathrm{II}}^{-1} A_{\mathrm{I} \Gamma}$. Let the $n_{\Gamma}$ eigenpairs $\left(\lambda_{\ell}^{(j)}, \mathbf{V}_{\Gamma, \ell}\right)_{\ell=1}^{n_{\Gamma}}$ corresponding to (3.7) be numbered in increasing order of $\lambda_{\ell}^{(j)}$. Since matrices $S_{\Gamma}$ and $M_{\Gamma}$ are symmetric the eigenvectors $\mathbf{V}_{\Gamma, \ell}, \ell=1, \ldots, n_{\Gamma}$, are orthogonal in the $S_{\Gamma}$ and in the $M_{\Gamma}$ 
inner products. Note that $S_{\Gamma}$ is symmetric positive semidefinite and that in the case of an interior subdomain, there is exactly one eigenvalue that is 0 with constant eigenvector.

Note that

$$
A^{(j)} \mathbf{V}_{\ell}^{(j)}=\lambda_{\ell}^{(j)}\left[\begin{array}{cc}
0 & 0 \\
0 & M_{\Gamma}
\end{array}\right] \mathbf{V}_{\ell}^{(j)}=M \mathbf{V}_{\ell}^{(j)}
$$

and so $\mathbf{V}_{\ell}^{(j)}$ is an eigenvector corresponding to the eigenvalue $\lambda_{\ell}^{(j)}$ of generalized eigenproblem (3.5). Moreover, since

$$
\left(\mathbf{V}_{k}^{(j)}\right)^{T} A^{(j)} \mathbf{V}_{\ell}^{(j)}=\left[\begin{array}{c}
-A_{\mathrm{II}}^{-1} A_{\mathrm{I} \Gamma} \mathbf{V}_{\Gamma, k} \\
\mathbf{V}_{\Gamma, k}
\end{array}\right]^{T}\left[\begin{array}{c}
\mathbf{0} \\
S_{\Gamma} \mathbf{V}_{\Gamma, \ell}
\end{array}\right]=\mathbf{V}_{\Gamma, k}^{T} S_{\Gamma} \mathbf{V}_{\Gamma, \ell}=0
$$

the vectors $\left\{\mathbf{V}_{\ell}^{(j)}\right\}_{\ell=1}^{n_{\Gamma}}$ are $A^{(j)}$-orthogonal. Let us assume further, that they are normalised in the $A^{(j)}$-norm, such that $\left\|\mathbf{V}_{\ell}^{(j)}\right\|_{A^{(j)}}=1$. Since $\left(A^{(j)}\right)^{-1}\left[\begin{array}{cc}0 & 0 \\ 0 & M_{\Gamma}\end{array}\right]$ has only got $n_{\Gamma}$ nonzero eigenvalues, all the remaining eigenvalues of (3.8) are $\infty$, and so the smallest eigenvalues in (3.7) are also the smallest eigenvalues of (3.8).

The local coarse space is now defined as the span of the finite element functions $v_{\ell}^{(j)} \in$ $V_{h}\left(\Omega_{j}\right), \ell \leq m_{j} \leq n_{\Gamma}$, corresponding to the first $m_{j}$ eigenpairs of (3.8). For any $u \in V_{h}\left(\Omega_{j}\right)$, we can define the projection on $\operatorname{span}\left\{v_{\ell}^{(j)}\right\}_{\ell=1}^{m_{j}}$ by

$$
\Pi_{j} u:=\sum_{\ell=1}^{m_{j}} a_{j}\left(v_{\ell}^{(j)}, u\right) v_{\ell}^{(j)}
$$

which is stable and satisfies a weak approximation property, as the following theorem shows.

Theorem 3.2. Let Assumption 2.1 hold. Then, for any $u \in V_{h}\left(\Omega_{j}\right)$,

$$
\begin{aligned}
\left|\Pi_{j} u\right|_{a, \Omega_{j}} & \leq|u|_{a, \Omega_{j}} \text { and } \\
\left\|u-\Pi_{j} u\right\|_{0, \alpha, \Omega_{j}^{\circ}} & \lesssim \sqrt{c_{j}\left(m_{j}\right)} \delta_{j}|u|_{a, \Omega_{j}},
\end{aligned}
$$

where $c_{j}\left(m_{j}\right):=C_{P}^{2}+\left(\delta_{j} \lambda_{m_{j}+1}^{(j)}\right)^{-1}$.

Proof. The stability estimate (3.10) follows immediately from the fact that $\Pi_{j}$ is an $a^{(j)_{-}}$ orthogonal projection. To prove (3.11) let us first apply Lemma 2.4, i.e.

$$
\left\|u-\Pi_{j} u\right\|_{0, \alpha, \Omega_{j}^{\circ}}^{2} \lesssim C_{P} \delta_{j}^{2}\left|u-\Pi_{j} u\right|_{a, \Omega_{j}^{\circ}}^{2}+\delta_{j}\left\|u-\Pi_{j} u\right\|_{0, \operatorname{tr}_{j} \alpha, \Gamma}^{2},
$$

It follows from the triangle inequality and (3.10) that

$$
\left|u-\Pi_{j} u\right|_{a, \Omega_{j}^{\circ}}^{2} \leq\left|u-\Pi_{j} u\right|_{a, \Omega_{j}}^{2} \lesssim|u|_{a, \Omega_{j}}^{2}
$$

and so it only remains to bound $\left\|u-\Pi_{j} u\right\|_{0, \operatorname{tr}_{j} \alpha, \Gamma}^{2}$.

To do this, let us extend the set $\left\{v_{\ell}^{(j)}\right\}_{\ell=1}^{n_{\Gamma}}$ to an $a_{j}$-orthonormal basis of $V_{h}\left(\Omega_{j}\right)$ by adding $n_{j}=\operatorname{dim} V_{h, 0}\left(\Omega_{j}\right)=\#$ I suitable functions $v_{n_{\Gamma}+\ell}^{(j)}, \ell=1, \ldots, n_{j}$, and write

$$
u=\sum_{\ell=1}^{n_{j}+n_{\Gamma}} a_{j}\left(v_{\ell}^{(j)}, u\right) v_{\ell}^{(j)}
$$




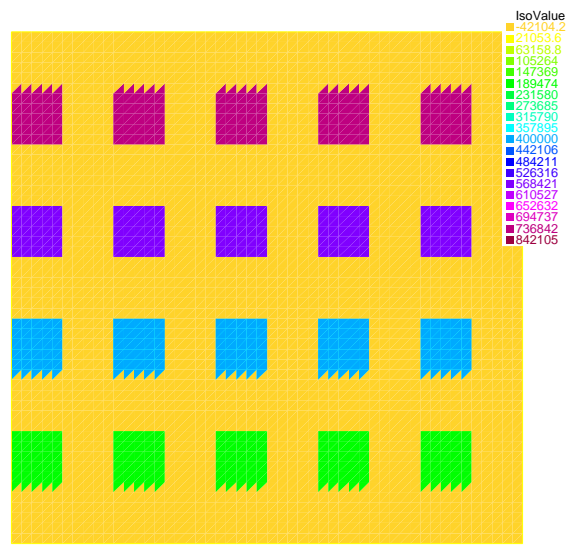

Figure 4. Coefficient distribution on a subdomain $\Omega_{j}$ for a two-dimensional model problem with high-permeability inclusions.

The restriction of the functions $\left\{v_{\ell}^{(j)}\right\}_{\ell=1}^{n_{\Gamma}}$ to the boundary $\Gamma$ forms a complete basis of $V_{h}(\Gamma)$. This implies that $v_{n_{\Gamma}+\ell}^{(j)} \equiv 0$ on $\Gamma$, for all $\ell=1, \ldots, n_{j}$. Moreover, it follows from the definition of the eigenproblem (3.7) that the functions $\left\{v_{\ell}^{(j)}\right\}_{\ell=1}^{n_{\Gamma}}$ are orthogonal also in the $(\cdot, \cdot)_{0, \operatorname{tr}_{j} \alpha, \Gamma}$ inner product. Therefore

$$
\begin{aligned}
\left\|u-\Pi_{j} u\right\|_{0, \operatorname{tr}_{j} \alpha, \Gamma}^{2} & =\sum_{\ell=m_{j}+1}^{n_{\Gamma}} a\left(v_{\ell}^{(j)}, u\right)^{2}\left\|v_{\ell}^{(j)}\right\|_{0, \operatorname{tr}_{j} \alpha, \Gamma}^{2}=\sum_{\ell=m_{j}+1}^{n_{\Gamma}} \frac{1}{\lambda_{\ell}^{(j)}} a\left(v_{\ell}^{(j)}, u\right)^{2} \\
& \leq \frac{1}{\lambda_{m_{j}+1}^{(j)}} \sum_{\ell=1}^{n_{\Gamma}} a\left(v_{\ell}^{(j)}, u\right)^{2}=\frac{1}{\lambda_{m_{j}+1}^{(j)}}|u|_{a, \Omega_{j}}^{2}
\end{aligned}
$$

and the result follows from (3.12), (3.13) and (3.15).

As explained in previous work (cf. [24]), we only include eigenvectors with eigenvalues smaller than $\operatorname{diam}\left(\Omega_{j}\right)^{-1}$ in the construction of the coarse space. In the constant coefficient case, the smallest eigenvalue of the DtN map is zero and it corresponds to the constant function 1. For a shape regular subdomain, the first positive eigenvalue is of $\operatorname{order} \operatorname{diam}\left(\Omega_{j}\right)^{-1}$, see [12]. In the heterogeneous case, due to the variation of the coefficients we may possibly have positive eigenvalues smaller than $\mathcal{O}\left(\operatorname{diam}\left(\Omega_{j}\right)^{-1}\right)$. Thus, in order to have a convergence behavior similar to that in the constant coefficient case, it is natural to keep all eigenvectors with eigenvalues smaller than $\operatorname{diam}\left(\Omega_{j}\right)^{-1}$.

Remark 3.3. We consider now the two-dimensional permeability field $\alpha$ of a subdomain shown in Figure 4. We show in Figure 5 a typical eigenvector used in [15] (left plot) and a typical generalized eigenvector introduced in our paper (right plot). We note that the eigenvectors used in the coarse space construction in the DtN case are related only to the high-permeability islands that cross the boundaries of the local domain. Whereas for the coarse space considered in [15] it is related to the islands in the interior of the subdomain and thus their number is substantially larger.

3.2. Global coarse space construction, stability and interpolation estimates. Using the partition of unity defined in Section 2.2, we now combine the local basis functions constructed in the previous section to obtain a coarse space $V_{H} \subset V_{h, 0}$ on all of $\Omega$. 

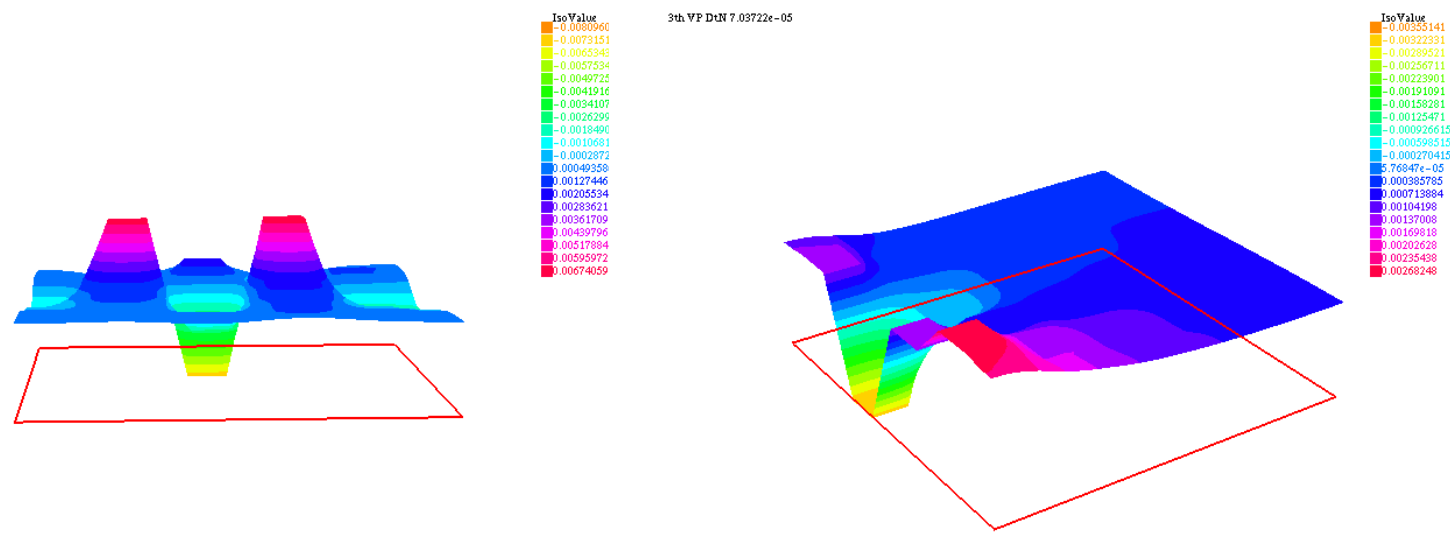

FiguRE 5. Eigenvector of the full subdomain eigenproblem for the model problem in Figure 4 used in the coarse space construction in [15] (left) and eigenvector of the interface-DtN map used in our construction here (right).

For any $\Omega_{j}, 1 \leq j \leq J$, let $\Pi_{j}$ be the projection onto the first $m_{j}$ local DtN eigenvectors defined in (3.9) and let $\chi_{j}$ be the partition of unity function associated with $\Omega_{j}$ defined in (2.7). The new coarse space is now defined as

$$
V_{H}:=\operatorname{span}\left\{\Phi_{j, \ell}^{H}: 1 \leq j \leq J \text { and } 1 \leq \ell \leq m_{j}\right\}, \quad \text { where } \quad \Phi_{j, \ell}^{H}:=I_{h}\left(\chi_{j} v_{\ell}^{(j)}\right)
$$

and $I_{h}$ is the standard nodal interpolant onto $V_{h, 0}(\Omega)$. The dimension of $V_{H}$ is $\sum_{j=1}^{J} m_{j}$. By construction each of the functions $\Phi_{j, \ell} \in V_{h_{0}}$, so that as required $V_{H} \subset V_{h, 0}$.

For a given function $u \in V_{h, 0}$, we introduce the coarse interpolant of $u$ as

$$
u_{0}:=I_{h}\left(\left.\sum_{j=1}^{J} \chi_{j} \Pi_{j} u\right|_{\Omega_{j}}\right) \in V_{H} .
$$

In the following, to ease the presentation when there is no confusion and it is clear from the context, we will simply denote the restriction $\left.u\right|_{\Omega_{j}}$ of $u$ onto $\Omega_{j}$ by $u$, and write, e.g., $\Pi_{j} u$ instead of $\left.\Pi_{j} u\right|_{\Omega_{j}}$.

The following theorem is key to proving the robustness of this coarse space construction.

Theorem 3.4. Let $u \in V_{h, 0}$ be given and let $u_{0} \in V_{H}$ be the coarse grid interpolant of $u$, defined in (3.16). Then there are functions $u_{j} \in V_{h, 0}\left(\Omega_{j}\right), j=1, \ldots, J$, such that

$$
u=\sum_{j=0}^{J} u_{j} \quad \text { and } \sum_{j=0}^{J}\left\|u_{j}\right\|_{a}^{2} \lesssim \max _{j=1}^{J}\left\{c_{j}\left(m_{j}\right)\right\}\|u\|_{a}^{2},
$$

i.e. there exists a stable splitting of $u$.

Proof. We choose $u_{j}:=I_{h}\left(\chi_{j}\left(u-\Pi_{j} u\right)\right)$. Then clearly, since by definition $u=\sum_{j=1}^{J} \chi_{j} u$,

$$
\sum_{j=1}^{J} u_{j}=\sum_{j=1}^{J} I_{h}\left(\chi_{j}\left(u-\Pi_{j} u\right)\right)=I_{h}\left(\sum_{j=1}^{J} \chi_{j} u\right)-\sum_{j=1}^{J} I_{h}\left(\chi_{j} \Pi_{j} u\right)=u-u_{0} .
$$


It follows from a simple application of the triangle inequality that

$$
\sum_{j=0}^{J}\left\|u_{j}\right\|_{a}^{2} \leq\left\|u-u_{0}\right\|_{a}^{2}+\|u\|_{a}^{2}+\sum_{j=1}^{J}\left\|u_{j}\right\|_{a}^{2}
$$

Since the interpolant $I_{h}$ is stable with respect to the $a$-norm (cf. [33, Lemma 2.3]) and since each point is overlapped by at most $N_{0}$ domains, we have

$$
\left\|u-u_{0}\right\|_{a}^{2}=\left\|I_{h}\left(u-\sum_{j=1}^{J} \chi_{j} \Pi_{j} u\right)\right\|_{a}^{2} \lesssim\left\|u-\sum_{j=1}^{J} \chi_{j} \Pi_{j} u\right\|_{a}^{2} \lesssim \sum_{j=1}^{J}\left\|\chi_{j}\left(u-\Pi_{j} u\right)\right\|_{a}^{2}
$$

Substituting this into (3.17) and using the definition of $u_{j}$ as well as the $a$-stability of the interpolant $I_{h}$ we get

$$
\sum_{j=0}^{J}\left\|u_{j}\right\|_{a}^{2} \lesssim \sum_{j=1}^{J}\left\|\chi_{j}\left(u-\Pi_{j} u\right)\right\|_{a}^{2}+\|u\|_{a}^{2}
$$

Note that $\operatorname{supp}\left\{\chi_{j}\right\}=\bar{\Omega}_{j}$ and $\operatorname{supp}\left\{\nabla \chi_{j}\right\}=\bar{\Omega}_{j}^{\circ}$. Thus, using triangle inequality and product rule

$$
\begin{aligned}
\sum_{j=1}^{J}\left\|\chi_{j}\left(u-\Pi_{j} u\right)\right\|_{a}^{2} & \lesssim \sum_{j=1}^{J}\left\|\chi_{j}\right\|_{\infty}^{2}\left|u-\Pi_{j} u\right|_{a, \Omega_{j}}^{2}+\left\|\nabla \chi_{j}\right\|_{\infty}^{2}\left\|u-\Pi_{j} u\right\|_{0, \alpha, \Omega_{j}^{\circ}}^{2} \\
& \lesssim \sum_{j=1}^{J}|u|_{a, \Omega_{j}}^{2}+\left|\Pi_{j} u\right|_{a, \Omega_{j}}^{2}+\delta_{j}^{-2}\left\|u-\Pi_{j} u\right\|_{0, \alpha, \Omega_{j}^{\circ}}^{2} .
\end{aligned}
$$

Note that in the last part we used the property of the partition of unity (2.8).

Since it was assumed that each point $\boldsymbol{x} \in \Omega$ is contained in at most $N_{0}$ subdomains, we can now use Theorem 3.2 to deduce from (3.18) and (3.19) that

$$
\sum_{j=0}^{J}\left\|u_{j}\right\|_{a}^{2} \lesssim\|u\|_{a}^{2}+\sum_{j=1}^{J}|u|_{a, \Omega_{j}}^{2} c_{j}\left(m_{j}\right) \lesssim \max _{j=1}^{J}\left\{c_{j}\left(m_{j}\right)\right\}\|u\|_{a}^{2},
$$

which ends the proof.

As usual (see e.g. [34]), the existence of a stable splitting established in Theorem 3.4 together with the hypothesis that each point $\boldsymbol{x} \in \Omega$ is contained in at most $N_{0}$ subdomains is sufficient to deduce the following bound on the condition number of $M_{A S, 2}^{-1} A$ from the abstract Schwarz theory.

Theorem 3.5. Let Assumption 2.1 be satisfied. Then the condition number of the two-level Schwarz algorithm with a coarse space based on local DtN maps can be bounded by

$$
\kappa\left(M_{A S, 2}^{-1} A\right) \lesssim \max _{j=1}^{J}\left\{c_{j}\left(m_{j}\right)\right\} \lesssim\left(C_{P}^{2}+\max _{j=1}^{J} \frac{1}{\delta_{j} \lambda_{m_{j}+1}^{(j)}}\right) .
$$

The hidden constant is independent of $h, \delta_{j}$, and $\operatorname{diam}\left(\Omega_{j}\right)$, as well as any jumps in $\alpha$. 


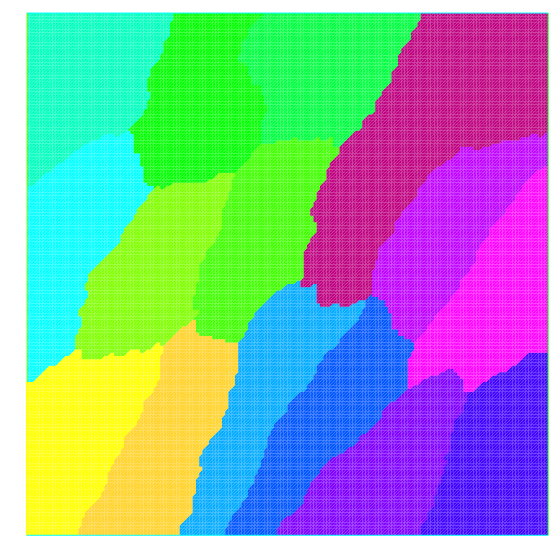

FiguRE 6. Subdomain partitioning into 16 subdomains using METIS.

Remark 3.6. Note that, by choosing the number $m_{j}$ of modes per subdomain in the coarse space as discussed at the end of Section 3.1, i.e. such that $\lambda_{m_{j}+1}^{(j)} \geq \operatorname{diam}\left(\Omega_{j}\right)^{-1}$, the method we implement in the next section satisfies

$$
\kappa\left(M_{A S, 1}^{-1} A\right) \lesssim\left(C_{P}^{2}+\max _{j=1}^{J} \frac{\operatorname{diam}\left(\Omega_{j}\right)}{\delta_{j}}\right)
$$

Hence, provided the weighted Poincaré constant $C_{P}$ in Assumption 2.1 is uniformly bounded, independently of any jumps in the coefficients, we retrieve the classical estimate for the Additive Schwarz Method. An interesting observation is that the bound depends only in an additive way on the constant $C_{P}$ and on the ratio of subdomain diameter to overlap.

\section{Numerical Results}

We solve the model problem (2.1) on the domain $\Omega=[0,1]^{2}$ using standard continuous, piecewise linear $\left(P_{1}\right)$ finite elements. The diffusion $\alpha$ is a function of $\boldsymbol{x}$. The boundary condition is $u=0$ on the left side boundary and $\frac{\partial u}{\partial n}=0$ on the remainder. The corresponding discretizations and data structures were obtained by using the software FreeFem $++[18]$ in connection with the METIS partitioner [19]. We will test the standard additive Schwarz (AS) and the restricted additive Schwarz (RAS) preconditioners with and without coarse space, in particular comparing the new coarse space based on harmonic extensions of eigenvectors of the local DtN operators with the standard coarse space that is the piecewise constant space of Nicolaides [25]. We test the method on (fairly irregular) overlapping partitions into $J$ subdomains. These overlapping partitions are built by adding layers to non-overlapping ones obtained, e.g., via graph partitioner METIS (see Figure 6). Extensive numerical results have been presented in a previous paper [24]. Here we present only a selection of difficult test cases, with so called inclusions and channels.

In order to compare our method to existing codes we solve two test cases with known difficulties. The diffusion coefficient $\alpha$ is highly heterogeneous, it takes values between 1 and approximately $2 \times 10^{6}$ and contains both high-permeability inclusions and channels. First of all we will analyze the performance of the method by increasing the number of channels and then by increasing the number of inclusions. 

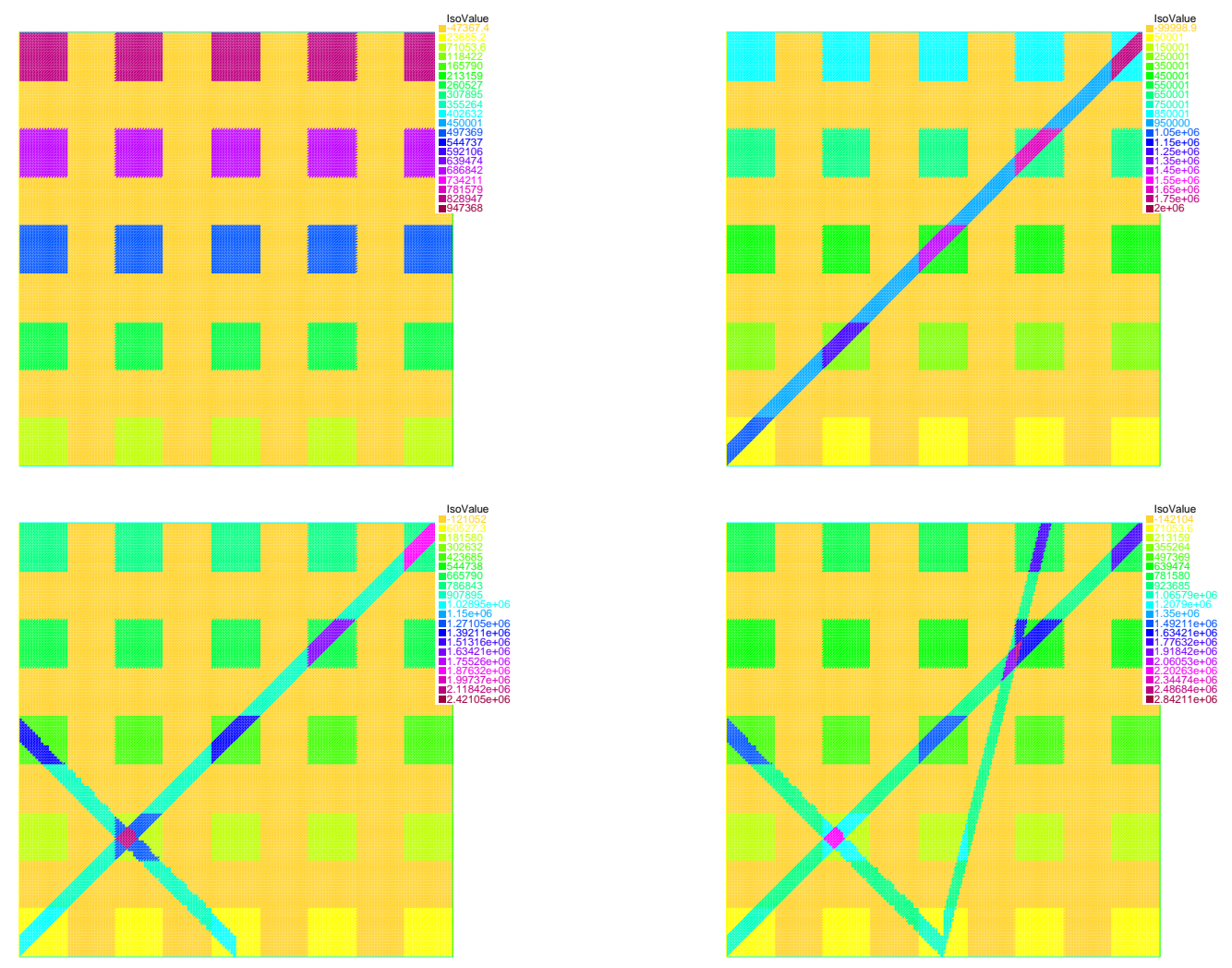

Figure 7. Test Problem 1: Successively adding channels.

\begin{tabular}{|c||c|c|c||c|c|c|}
\hline & AS & AS+Nicolaides & AS+DtN & RAS & RAS+Nicolaides & RAS+DtN \\
\hline no channel & 529 & 1000 & 57 & 243 & 245 & 41 \\
1 channel & 619 & 520 & 64 & 227 & 228 & 46 \\
2 channels & $>1000$ & 516 & 68 & 226 & 226 & 47 \\
3 channels & 585 & 697 & 76 & 212 & 213 & 44 \\
\hline
\end{tabular}

TABLE 1. Number of iterations for Test Problem 1 (additive coarse grid correction).

We use a uniform triangulation with $160 \times 160$ nodes and a partition into 16 (irregular) subdomains (see Figure 6). Each subdomain is extended by one layer, leading to an overlap of 2 layers, such that $\delta_{j}=2 h$ for all $j=1, \ldots, J$. . We use the AS preconditioner within conjugate gradients (CG) and the RAS preconditioner within GMRES, and in each case we stop the iteration process, when the relative residual is smaller than $10^{-6}$.

We start with only inclusions and add the channels one by one as shown in Figure 7 (Test Problem 1). When there are no channels, $\alpha$ varies between 1 and $10^{6}$, as indicated by the colours in Figure 7. With all three channels present, $\alpha$ varies between 1 and $2.8 \times 10^{6}$. The corresponding convergence results are given in Table 1. Our algorithm performs significantly better. The piecewise constant coarse space has virtually no effect on the performance of either AS or RAS, leading to iteration numbers that differ little from the results without any coarse grid in all four cases. Our new coarse space, on the other hand, is fully robust 


\begin{tabular}{|c||c|c|c||c|c|c|}
\hline & AS & AS+Nicolaides & AS+DtN & RAS & RAS+Nicolaides & RAS+DtN \\
\hline no channel & 529 & 656 & 39 & 243 & 231 & 25 \\
1 channel & 619 & 538 & 41 & 227 & 215 & 28 \\
2 channels & $>1000$ & 808 & 47 & 226 & 211 & 27 \\
3 channels & 585 & 641 & 47 & 212 & 199 & 28 \\
\hline
\end{tabular}

TABle 2. Number of iterations for Test Problem 1 (deflation-based coarse grid correction).

\begin{tabular}{|c||c||c|c|c|c|}
\hline \multicolumn{1}{|c||}{$\begin{array}{c}\text { Over } J=16 \\
\text { subdomains }\end{array}$} & \multicolumn{2}{c||}{$\begin{array}{c}\text { Total number } n_{\Gamma j} \text { of } \\
\text { eigenvalues on } \Gamma_{j}\end{array}$} & \multicolumn{4}{c|}{ Number $m_{j}$ of functions included in $V_{H}$ from $\Omega_{j}$} \\
\hline \hline Minimum & 70 & 1 & 1 & 1 & 1 \\
\hline Maximum & 191 & 4 & 4 & 4 & 4 \\
\hline Average & 138.8 & 2.75 & 2.88 & 2.94 & 2.94 \\
\hline \hline Sum & 2220 & 44 & 46 & 47 & 47 \\
\hline
\end{tabular}

TABLE 3. Size of the coarse space for Test Problem 1.

to the coefficient variation and to the addition of channels, and it leads to a gain of at least a factor 8 compared to the one-level method in all cases. The situation is similar, if we use deflation-based coarse grid correction [22] with the same coarse spaces (see Table 2). However, the absolute numbers of iterations are reduced almost by a factor 2 in this case. Our theory applies equally to this case (see e.g. [17] for details), but we will not include any further numerical results with deflation-based coarse grid correction.

Table 3 gives some information on the size of the coarse space that we build with our automatic selection strategy: for each number of channels we give $\min _{j} m_{j}$ and $\max _{j} m_{j}$, as well as the global coarse space size $n_{H}=\sum_{j} m_{j}$ and the average number of modes included per subdomain $n_{H} / J$. For comparison, we also include information on the total number $n_{\Gamma j}$ of eigenmodes of the discrete DtN operator on each subdomain. We note that adding channels does not have a big influence on the size of the coarse space; we only need three additional eigenvectors in the case of three channels compared to the case of no channels.

Then, using the same domain and the same partition we successively add inclusions without any channels present as shown in Figure 8 (Test Problem 2). The results are in Table 4. Again, the piecewise constant coarse space is not working at all for this test problem. The DtN-based coarse space is almost completely robust to an increase in the number of inclusions and requires again significantly less iterations than the one-level method inall cases. Note that the subdomain partition (cf. Figure 6) is not aligned with the inclusions at all (cf. Figure 8). In Table 5 we see that also in this test problem, the coarse space size grows only very slowly with the number of inclusions and even in the hardest case $n_{H}$ is only 53 (cf. the dimension $n$ of $V_{h, 0}$, and thus of $A$ is 25600).

The last series of tests, in Table 6 , aims to prove that the number $m_{j}$ of eigenvectors per subdomain chosen by our automatic algorithm is indeed optimal in some sense. For Test Problem 1 with one channel, we first reduce the number of coarse basis functions per subdomain by one, this has a huge influence on the iteration count. Then we add one basis 

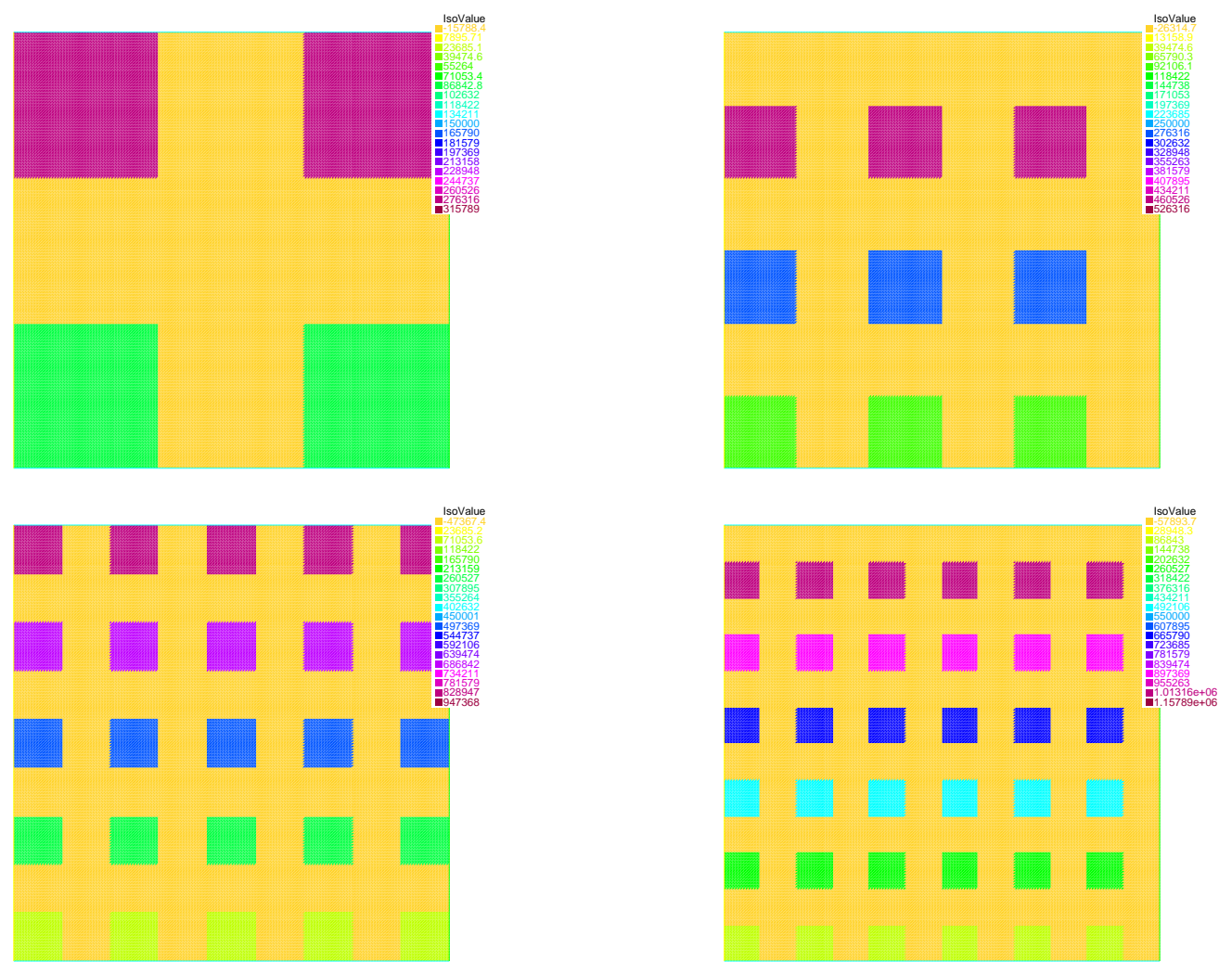

Figure 8. Test Problem 2: Successively adding inclusions.

\begin{tabular}{|c||c|c|c||c|c|c|}
\hline & AS & AS+Nicolaides & AS+DtN & RAS & RAS+Nicolaides & RAS+DtN \\
\hline $2 \times 2$ inclusions & 108 & 80 & 51 & 100 & 81 & 41 \\
\hline $3 \times 3$ inclusions & 194 & 342 & 58 & 154 & 153 & 46 \\
\hline $5 \times 5$ inclusions & 529 & $>1000$ & 57 & 243 & 245 & 41 \\
\hline $6 \times 6$ inclusions & 835 & 823 & 71 & 266 & 267 & 51 \\
\hline
\end{tabular}

TABLE 4. Number of iterations for Test Problem 2 (additive coarse grid correction).

\begin{tabular}{|c||c||c|c|c|c|}
\hline \multicolumn{1}{|c||}{ Over $J=16$} & \multicolumn{1}{c||}{ Total number $n_{\Gamma j}$ of } & \multicolumn{4}{c|}{ Number $m_{j}$ of functions included in $V_{H}$ from $\Omega_{j}$} \\
subdomains & eigenvalues on $\Gamma_{j}$ & no channel & 1 channel & 2 channels & 3 channels \\
\hline \hline Minimum & 70 & 1 & 1 & 1 & 1 \\
\hline Maximum & 191 & 3 & 3 & 4 & 5 \\
\hline Average & 138.8 & 1.63 & 2.06 & 2.75 & 3.31 \\
\hline \hline Sum & 2220 & 26 & 33 & 44 & 53 \\
\hline
\end{tabular}

TABle 5. Size of the coarse space for Test Problem 2. 
function per subdomain and notice that this has much less effect. This suggests that the selection process we have designed is indeed the best compromise between enriching the coarse grid and solving a reasonably sized coarse problem.

\begin{tabular}{|l|c|c|}
\hline & AS & RAS \\
\hline No coarse space & 619 & 227 \\
Nicolaides' piecewise constant coarse space & 520 & 228 \\
DtN space with $\max \left\{m_{j}-1,1\right\}$ functions from $\Omega_{j}$ & 446 & 177 \\
DtN space with $m_{j}$ functions from $\Omega_{j}$ & 64 & 46 \\
DtN space with $m_{j}+1$ functions from $\Omega_{j}$ & 37 & 32 \\
\hline
\end{tabular}

TABLE 6. Iteration numbers when reducing or increasing the number $m_{j}$ of coarse basis functions per subdomain given by the automatic selection strategy.

\section{Conclusions}

In this paper we have given a rigorous analysis of the coarse space proposed in [23, 24]. We have seen that a robust coarse space can be chosen automatically with the method proposed there, if all the eigenmodes of local DtN maps that are smaller than the inverse of the diameter of the respective subdomain are included in the coarse space. Moreover, our results show that this selection criterion is to some extent optimal with respect to cost and robustness. It remains to be seen whether the proposed coarse space has also got good approximation properties in the context of multiscale approximation techniques from problems, where it is not desirable to resolve all the fine scale variation. For the coarse space based on subdomain eigenproblems developed in the context of two-level Schwarz methods in [15], this has already been demonstrated to some extent in [11]. We are also currently working on the extension of the ideas in this paper to linear elasticity problems.

We included a small number of numerical tests to confirm the theoretical results on some hard test problems with a complicated coefficient distribution (channels and inclusions) and a high contrast in the values of the coefficient. We did not study the dependence on geometrical parameters in detail, and because we only considered small overlap it was also not possible to verify how important Assumption 2.1 is in practice. Figure 2 gives typical situations where the assumption is not satisfied, but we believe that in practice a much more complicated coefficient distribution and a larger overlap would be necessary to really observe any loss of robustness of the proposed method. The extensive tests in $[23,24]$ do also suggest this.

\section{REFERENCES}

1. J. H. Bramble, J. E. Pasciak, and A. H. Schatz, The construction of preconditioners for elliptic problems by substructuring. I, Math. Comp. 47 (1986), no. 175, 103-134. MR 842125 (87m:65174)

2. M. Brezina, C. Heberton, J. Mandel, and P. Vaněk, An iterative method with convergence rate chosen a priori, Tech. Report 140, University of Colorado Denver, CCM, University of Colorado Denver, April 1999, Earlier version presented at 1998 Copper Mountain Conference on Iterative Methods, April 1998.

3. Xiao-Chuan Cai and Marcus Sarkis, A restricted additive Schwarz preconditioner for general sparse linear systems, SIAM J. Sci. Comput. 21 (1999), no. 2, 792-797 (electronic). MR 1718707 (2000f:65133)

4. T. Chartier, R. D. Falgout, V. E. Henson, J. Jones, T. Manteuffel, S. McCormick, J. Ruge, and P. S. Vassilevski, Spectral AMGe ( $\rho A M G e)$, SIAM J. Sci. Comput. 25 (2003), no. 1, 1-26. MR 2047193 (2004m:65036) 
5. C. Chevalier and F. Pellegrini, PT-SCOTCH: a tool for efficient parallel graph ordering, Parallel Computing 6-8 (2008), no. 34, 318-331.

6. C. R. Dohrmann and O. B. Widlund, An overlapping Schwarz algorithm for almost incompressible elasticity, SIAM J. Numer. Anal. 47 (2009), no. 4, 2897-2923. MR 2551151 (2010i:65264)

7. Hybrid domain decomposition algorithms for compressible and almost incompressible elasticity, Internat. J. Numer. Methods Engrg. 82 (2010), no. 2, 157-183. MR 2640680

8. M. Dryja, M. V. Sarkis, and O. B. Widlund, Multilevel Schwarz methods for elliptic problems with discontinuous coefficients in three dimensions, Numer. Math. 72 (1996), no. 3, 313-348. MR 1367653 (96h:65134)

9. M. Dryja and O. B. Widlund, Some domain decomposition algorithms for elliptic problems, Iterative methods for large linear systems (L. Hayes and D. Kincaid, eds.), Academic Press, 1989, pp. $273-291$.

10. Y. Efendiev, J. Galvis, and P. S. Vassilevski, Spectral element agglomerate algebraic multigrid methods for elliptic problems with high contrast coefficients, Domain Decomposition Methods in Science and Engineering XIX (Berlin) (Y. Huang, R. Kornhuber, O. Widlund, and J. Xu, eds.), Lecture Notes in Computational Science and Engineering, vol. 78, Springer, 2011, pp. 407-414.

11. Y. Efendiev, J. Galvis, and X.-H. Wu, Multiscale finite element methods for high-contrast problems using local spectral basis functions, Journal of Computational Physics 230 (2011), 937-955.

12. J. F. Escobar, The geometry of the first non-zero Stekloff eigenvalue, J. Funct. Anal. 150 (1997), no. 2, 544-556. MR 1479552 (98g:58180)

13. C. Farhat and F.-X. Roux, A method of finite element tearing and interconnecting and its parallel solution algorithm, International Journal for Numerical Methods in Engineering 32 (1991), 1205-1227.

14. J. Galvis and Y. Efendiev, Domain decomposition preconditioners for multiscale flows in high-contrast media, Multiscale Model. Simul. 8 (2010), no. 4, 1461-1483. MR 2718268

15. _ Domain decomposition preconditioners for multiscale flows in high contrast media: Reduced dimension coarse spaces, Multiscale Modeling \& Simulation 8 (2010), no. 5, 1621-1644.

16. I. G. Graham, P. O. Lechner, and R. Scheichl, Domain decomposition for multiscale PDEs, Numer. Math. 106 (2007), no. 4, 589-626. MR MR2317926 (2008f:65242)

17. I. G. Graham and Scheichl R., Robust domain decomposition algorithms for multiscale PDEs, Numerical Methods for Partial Differential Equations 23 (2007), no. 4, 859-878.

18. Frédéric Hecht, Freefem ++ , 3.7 ed., Numerical Mathematics and Scientific Computation, Laboratoire J.L. Lions, Université Pierre et Marie Curie, http://www.freefem.org/ff++/, 2010.

19. G. Karypis and V. Kumar, METIS: A software package for partitioning unstructured graphs, partitioning meshes, and computing fill-reducing orderings of sparse matrices, Tech. report, Department of Computer Science, University of Minnesota, 1998, http://glaros.dtc.umn.edu/gkhome/views/metis.

20. J. Mandel, Balancing domain decomposition, Comm. Numer. Methods Engrg. 9 (1993), no. 3, $233-241$. MR 1208381 (94b:65158)

21. J. Mandel and M. Brezina, Balancing domain decomposition for problems with large jumps in coefficients, Math. Comp. 65 (1996), no. 216, 1387-1401. MR 1351204 (97a:65109)

22. R. Nabben and C. Vuik, A comparison of deflation and coarse grid correction applied to porous media flow, SIAM Journal on Numerical Analysis 42 (2004), 1631-1647.

23. F. Nataf, H. Xiang, and V. Dolean, A two level domain decomposition preconditioner based on local Dirichlet-to-Neumann maps, C. R. Mathématique 348 (2010), no. 21-22, 1163-1167.

24. F. Nataf, H. Xiang, V. Dolean, and N. Spillane, A coarse space construction based on local Dirichlet to Neumann maps, SIAM J. Sci Comput. (2011).

25. R. A. Nicolaides, Deflation of conjugate gradients with applications to boundary value problems, SIAM J. Numer. Anal. 24 (1987), no. 2, 355-365. MR 881370 (88h:65016)

26. C. Pechstein and R. Scheichl, Analysis of FETI methods for multiscale PDEs, Numer. Math. 111 (2008), no. 2, 293-333. MR 2456834 (2010g:65218)

27. Analysis of FETI methods for multiscale PDEs - Part II: Interface variation, Tech. Report BICS 7/09, Bath Institute for Complex Systems (BICS), BICS, University of Bath, UK, July 2009.

28. __ Scaling up through domain decomposition, Appl. Anal. 88 (2009), no. 10-11, 1589-1608. MR 2582178

29. __ Weighted Poincaré inequalities, Tech. Report NuMa-Report 2010-10, Institute of Computational Mathematics, Johannes Kepler University, Linz, December 2010, submitted. 
30. Weighted Poincaré inequalities and applications in domain decomposition, Domain Decomposition Methods in Science and Engineering XIX (Y. Huang, R. Kornhuber, O. Widlund, and J. Xu, eds.), Lecture Notes in Computational Science and Engineering, vol. 78, Springer, 2011, pp. 197-204. Also available as NuMa-Report 2009-12, Inst. Comput. Math., J.K. University Linz, November 2009.

31. R. Scheichl and E. Vainikko, Additive Schwarz with aggregation-based coarsening for elliptic problems with highly variable coefficients, Computing 80 (2007), no. 4, 319-343. MR MR2336234 (2008i:65293)

32. R. Scheichl, P. S. Vassilevski, and L. T. Zikatanov, Multilevel methods for elliptic problems with highly varying coefficients on non-aligned coarse grids, Tech. Report LLNL-JRNL-404462, Lawrence Livermore National Lab, August 2010, submitted to SINUM.

33. W Weak approximation properties of elliptic projections with functional constraints, Tech. Report LLNL-JRNL-462079, Lawrence Livermore National Lab, January 2011.

34. A. Toselli and O. B. Widlund, Domain decomposition methods - algorithms and theory, Springer Series in Computational Mathematics, vol. 34, Springer-Verlag, Berlin, 2005. MR 2104179 (2005g:65006)

35. J. Van lent, R. Scheichl, and I. G. Graham, Energy-minimizing coarse spaces for two-level Schwarz methods for multiscale PDEs, Numer. Linear Algebra Appl. 16 (2009), no. 10, 775-799. MR 2567313

Laboratoire J.-A.. Dieudonné, CNRS UMR 6621, Université de Nice-Sophia Antipolis,06108 Nice Cedex 02, France.

E-mail address: dolean@unice.fr

Laboratoire J.L. Lions, CNRS UMr 7598, Université Pierre et Marie Curie, 75005 Paris, FRANCE.

E-mail address: nataf@ann.jussieu.fr

Department of Mathematical Sciences, University of Bath, Bath BA2 7AY, UK.

E-mail address: R.Scheichl@bath.ac.uk

Laboratoire J.L. Lions, CNRS UMR 7598, Université Pierre et Marie Curie, 75005 Paris, FRANCE.

E-mail address: spillane@ann.jussieu.fr 